\title{
Modulation of dendritic cell function by Trichomonas vaginalis-derived secretory products
}

\author{
Min-Ji Song ${ }^{1}$, Jong-Joo Lee ${ }^{1,2}$, Young Hee Nam ${ }^{1}$, Tae-Gyun Kim ${ }^{1,2}$, Youn Wook Chung ${ }^{1}$, Mikyoung Kim ${ }^{1}$, Ye-Eun Choi ${ }^{1,2}$, \\ Myeong Heon Shin ${ }^{1}$ \& Hyoung-Pyo Kim ${ }^{1, *}$ \\ ${ }^{1}$ Department of Environmental Medical Biology, Institute of Tropical Medicine, Yonsei University College of Medicine, \\ ${ }^{2}$ Brain Korea 21 PLUS Project for Medical Science, Yonsei University College of Medicine, Seoul 120-752, Korea
}

Trichomoniasis caused by the parasitic protozoan Trichomonas vaginalis is the most common sexually transmitted disease in the world. Dendritic cells are antigen presenting cells that initiate immune responses by directing the activation and differentiation of naïve T cells. In this study, we analyzed the effect of Trichomonas vaginalis-derived Secretory Products on the differentiation and function of dendritic cells. Differentiation of bone marrow-derived dendritic cells in the presence of T. vaginalis-derived Secretory Products resulted in inhibition of lipopolysaccharide-induced maturation of dendritic cells, down-regulation of IL-12, and up-regulation of IL-10. The protein components of $T$. vaginalis-derived Secretory Products were shown to be responsible for altered function of bone marrow-derived dendritic cells. Chromatin immunoprecipitation assay demonstrated that IL-12 expression was regulated at the chromatin level in $T$. vaginalis-derived Secretory Productstreated dendritic cells. Our results demonstrated that $T$. vaginalis-derived Secretory Products modulate the maturation and cytokine production of dendritic cells leading to immune tolerance. [BMB Reports 2015; 48(2): 103-108]

\section{INTRODUCTION}

Dendritic cells (DCs) are professional antigen-presenting cells that provide a pivotal link between the external environment and the adaptive immune response (1). Maturation and activation of DCs involve enhanced expression of several molecules that play key roles in antigen presentation and co-stimulation such as major histocompatibility complex II (MHC-II), CD80, CD86, and CD40, in concert with the release of cytokines that influence the type and intensity of the immune responses (2).

*Corresponding author. Tel: +82-2-2228-1842; Fax: +82-2-363-8676; E-mail: kimhp@yuhs.ac

http://dx.doi.org/10.5483/BMBRep.2015.48.2.116

Received 29 May 2014, Revised 17 June 2014, Accepted 22 June 2014

Keywords: Chromatin, Cytokine, Dendritic cells, Tolerance, Trichomonas vaginalis
While the immune responses induced by DCs are critical to control and eliminate the invading pathogens, DCs are equally responsible for the maintenance of immune tolerance to prevent destructive autoimmune reactions against healthy tissues (3). In addition, reports have demonstrated that parasites can interfere with DC activity in order to promote a permissive environment for survival inside their host (4).

Trichomonas vaginalis is an extracellular flagellated protozoan parasite that infects the squamous epithelium of the genital tract of both females and males (5). It is responsible for trichomoniasis, one of the most common non-viral sexually transmitted diseases in the world (6). Typical symptoms in infected women include a purulent and frothy vaginal discharge accompanied by pruritus (7). Vaginal infection is associated with adverse outcomes of pregnancy (8), increased incidence of cervical cancer (9), and increased transmission of human immunodeficiency virus (HIV) (10).

Infection with $T$. vaginalis leads to an aggressive local cellular immune response with inflammation of the vaginal epithelium in women and the urethra of men $(11,12)$. T. vaginalis frequently causes punctate mucosal hemorrhages, which potentially compromise the mechanical barrier to infection (13). In addition, human neutrophils and macrophages stimulated with live $T$. vaginalis produce the chemokine IL-8 and the acute pro-inflammatory cytokines IL- 6 , TNF- $\alpha$ and IL-1 $\beta$ that are responsible for initiating the immune response to infection by recruiting immune cells $(14,15)$. However, the host immune response frequently fails to clear $T$. vaginalis, leading to chronic infection. There is evidence that $T$. vaginalis infection can induce a state of non-responsiveness to subsequent stimulation with bacterial lipopolysaccharide (LPS) (16). Contact of macrophages with $T$. vaginalis led to inhibition of proinflammatory cytokine production (by suppressing NF- $\mathrm{KB}$ activation (16)) and induction of immunosuppressive cytokine IL-10 production by human DCs (17). It also has been reported that $T$. vaginalis can induce macrophage apoptosis through phosphorylation of p38 mitogen-activated protein (MAP) kinase (18).

The pathogenicity of $T$. vaginalis is influenced by its contact with epithelial cells; however, various soluble factors secreted from $T$. vaginalis also have been shown to modulate host im- 
mune responses. Recent studies showed that $T$. vaginalis can secrete leukotriene $B_{4}$, which binds to Leukotriene $B_{4}$ receptors BLT1 and BLT2 on neutrophils and mast cells leading to production of IL-8 $(19,20)$. However, there is little information elucidating how $T$. vaginalis-derived Secretory Products (TvSP) affect the differentiation and maturation of DCs, which is critical given the crucial role of DCs in both the innate and adaptive immune responses.

In this study, we investigated the effect of TvSP on the surface molecule expression and cytokine secretion profile of DCs using an in vitro murine bone marrow-derived dendritic cell (BMDC) model.

\section{RESULTS}

\section{TvSP down-regulate the expression of MHC-II and} co-stimulatory molecules

To test whether TvSP affect differentiation and function of BMDC, bone marrow (BM) cells were cultured with granulocyte macrophage colony-stimulating factor (GM-CSF) in the presence or absence of TvSP for 10 days and then stimulated with LPS for $18 \mathrm{~h}$ to induce maturation of DCs. TvSP did not trigger apoptosis in BMDCs during GM-CSF-mediated differentiation (Supplementary Fig. 1). In the absence of TvSP, BM cells differentiated into DCs that displayed the typical surface phenotypes of immature and mature DCs (Fig. 1). When BM cells were differentiated in the presence of TVSP, however, the expression of MHC-II and co-stimulatory molecules such as CD40, CD80, and CD86 was down-regulated or at least remain unaltered in immature BMDCs (Fig. 1). The down-regulation of MHC-II and co-stimulatory molecules by TVSP was much more evident when DCs were stimulated with LPS (Fig. 1). These results suggest that TVSP impair the antigen-presenting capacity of DCs during DC differentiation by diminishing the expression of MHC-II and co-stimulatory molecules.

\section{Cytokine production by BMDC differentiated in the presence of TvSP}

In order to examine whether TvSP may affect the cytokine production capacity of BMDCs during DC differentiation, we performed quantitative real-time Polymerase Chain Reaction (qRT-PCR) using BMDCs that were differentiated in the absence or presence of TvSP for 10 days and then stimulated with LPS. This analysis demonstrated that the mRNA levels of $\|12 a\| 12 b,, \| 10$, Tnf, and $/ / 6$ were markedly increased by LPS stimulation in control BMDCs, consistent with a previous report (2). Interestingly, the induction of $I / 12 a$ and $I / 12 b$ mRNA expression in response to LPS was significantly lower in BMDCs differentiated in the presence of TVSP compared to the BMDCs differentiated in the absence of TvSP (Fig. 2A and 2B). In contrast, differentiation of BMDCs in the presence of TvSP resulted in higher expression of the immunosuppressive cytokine $/ / 10$ compared to the control BMDCs (Fig. 2C). TvSP exerted a minimal effect on the mRNA expression of other proin- flammatory cytokines such as Tnf and I/6 (Fig. 2D and 2E).

The effect of TvSP on the cytokine production profile of BMDCs also was analyzed at the protein level. The LPS-mediated induction of IL-12 was significantly diminished in TvSP-treated BMDCs (Fig. 2F), while production of IL-10 was

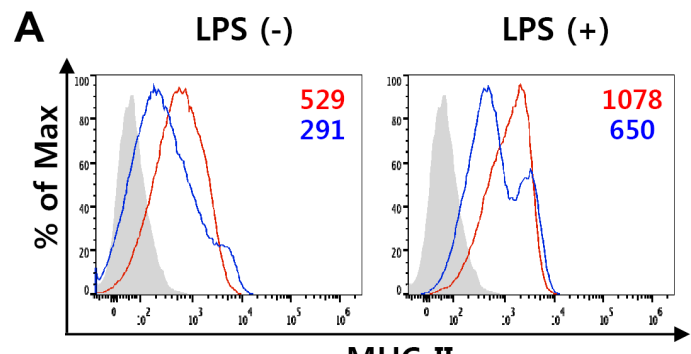

B

MHC-II

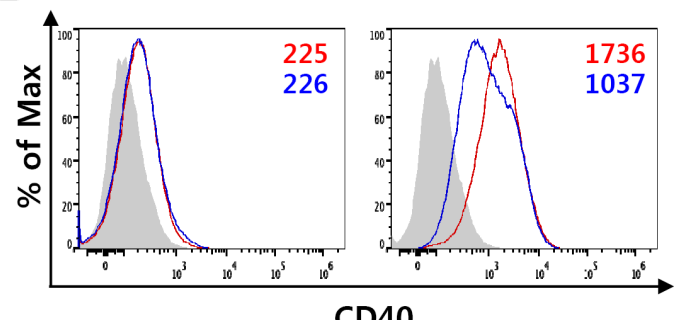

C

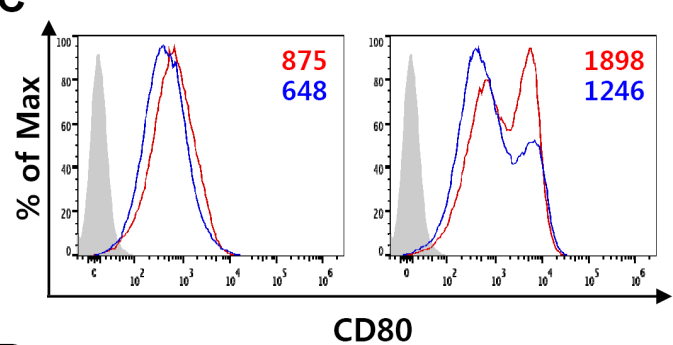

D

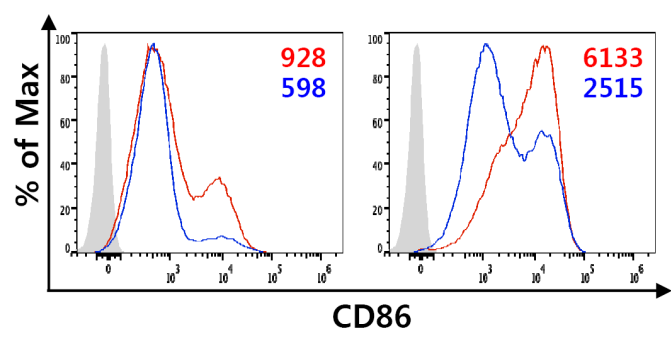

Fig. 1. The effect of TvSP on the expression of surface molecules on BMDC. DCs were differentiated with GM-CSF from BM precursors in the absence or presence of TvSP. On day 10, DCs were treated with $100 \mathrm{ng} / \mathrm{ml}$ LPS for $24 \mathrm{~h}$ to induce maturation, and expression of MHC-II (A), CD40 (B), CD80 (C) and CD86 (D) was analyzed by flow cytometry. Isotype controls are shown as shaded histograms, control BMDCs are shown as red lines, and TvSP-treated BMDCs are shown as blue lines. Mean fluorescent intensity (MFI) values are indicated with corresponding colors. Results are representative of more than three independent experiments. 

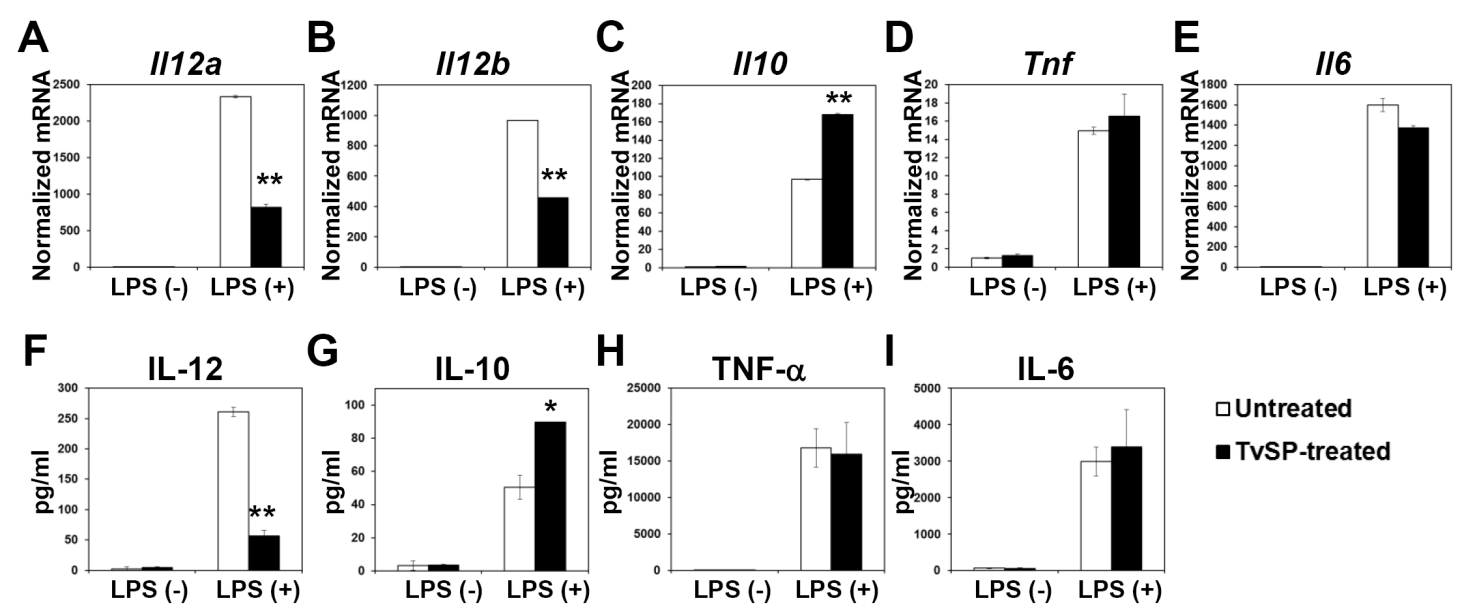

Fig. 2. The effect of TvSP on the cytokine production of BMDC. DCs were differentiated with GM-CSF from BM precursors in the absence or presence of TvSP. (A-E) Immature DCs were stimulated with $100 \mathrm{ng} / \mathrm{ml}$ LPS for $6 \mathrm{~h}$, and the mRNA levels of $/ / 12 a(A), / / 12 b$ (B), I/10 $(\mathrm{C})$, $\operatorname{Tnf}(\mathrm{D})$, and $/ / 6$ (E) were measured by qRT-PCR. (F-l) Immature DCs were stimulated with $100 \mathrm{ng} / \mathrm{ml}$ LPS for $24 \mathrm{~h}$, and the expression of IL-12 (F), IL-10 (G), TNF- $\alpha(\mathrm{H})$, and IL-6 (I) was measured by Cytometric Bead Array. All data are expressed as mean \pm SD, and statistical significance $\left({ }^{*} \mathrm{P}<0.05\right.$, or $\left.{ }^{* * P}<0.01\right)$ is shown for treatments compared with the controls.

increased by TvSP (Fig. 2G). The production of TNF- $\alpha$ and IL-6 were independent of TvSP treatment during BMDC differentiation (Fig. $2 \mathrm{H}$ and $2 \mathrm{l}$ ).

\section{The DC-modulatory activity of TvSP is abolished by treatment with Proteinase $\mathrm{K}$}

To understand the components of TvSP that are responsible for the regulation of surface molecule expression and cytokine production, we treated TvSP with Proteinase $\mathrm{K}$ and examined the effects on BMDCs. Compared to the mock-treated TvSP, which down-regulated expression of MHC-II, CD40, CD80, and CD86 especially when BMDCs were treated with LPS, treatment of TVSP with Proteinase $\mathrm{K}$ abrogated its inhibitory activity on the expression of these surface molecules (Fig. 3A-3D). The down-regulation of IL-12 production and up-regulation of IL-10 expression by TvSP also were abolished by Proteinase $\mathrm{K}$ treatment (Fig. 3E and 3F). These results show that a proteinaceous component of TvSP was responsible for the modulatory functions of BMDCs during differentiation with GM-CSF.

The effect of TvSP on the LPS-mediated signaling in BMDCs We next investigated the effect of TvSP on the signaling pathways activated by LPS in BMDCs. Three main families of MAP kinases including ERK, p38 MAPK, and JNK are reported to be involved in signal transduction during LPS-induced DC activation $(21,22)$. Phosphorylation of these kinases induced by LPS treatment in BMDCs that were differentiated in the presence of Proteinase K-treated PBS, mock-treated TvSP, and Proteinase K-treated TvSP was comparable to that in control BMDCs (Supplementary Fig. 2A). We also examined the effect of TvSP on NF-kB activation, but no significant difference was ob- served between BMDCs differentiated in the absence or presence of TvSP (Supplementary 2B). These data indicate that down-regulation of surface molecules and the altered cytokine production profile in BMDCs differentiated in the presence of TvSP may not be attributed to a defect in ERK, p38 MAPK, JNK, or NF-kB activation.

\section{The effect of TvSP on the chromatin structure of cytokine genes in BMDCs}

Regions of open chromatin are typically associated with transcriptionally permissive histone modifications, which are paralleled by accessibility to transcription factors (23). We performed chromatin immunoprecipitation (ChIP) assays to detect trimethyl-histone H3 Lys4 (H3K4me3), a histone modification specifically enriched at active or poised transcription start sites. Compared to the control BMDCs, enrichment of H3K4me3 was clearly reduced at the promoter region of the $1 / 12 \mathrm{a}$ gene in TvSP-treated BMDCs (Fig. 4A). In contrast, there were no significant differences in $\mathrm{H} 3 \mathrm{~K} 4 \mathrm{me} 3$ levels at the promoter regions of the $I / 12 b, \| 10, T n f, I / 6$, and Gapdh genes regardless of TvSP treatment (Fig. 4B-4F). These data demonstrated that transcriptional repression of the $/ 112 \mathrm{a}$ gene encoding IL-12p35 in TvSP-treated BMDCs takes place at the chromatin level and TvSP treatment during BMDCs differentiation altered the chromatin structure to be less accessible to the transcription factors required for gene expression.

\section{DISCUSSION}

In this study, we investigated the function of BMDCs that were exposed to soluble factors secreted from $T$. vaginalis during GM-CSF-mediated differentiation. Several lines of evidence 


\section{LPS $(-)$}

A

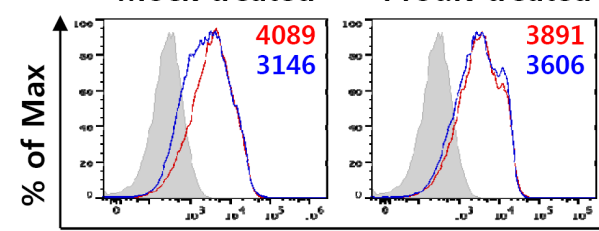

B
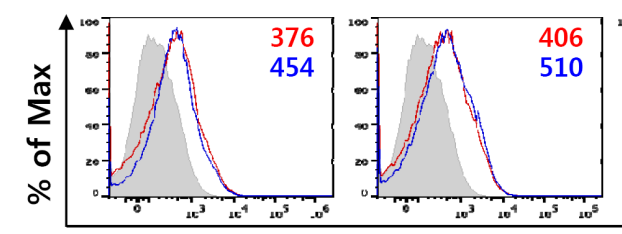

MHC-II

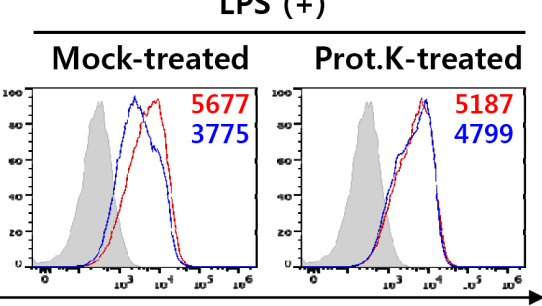

PS (+)

(10) 6439

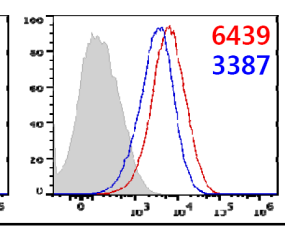

CD40
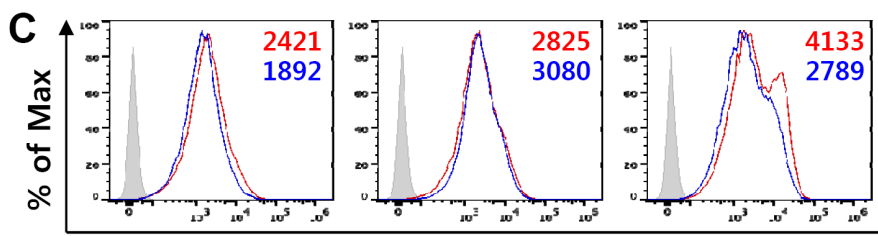

CD80

D
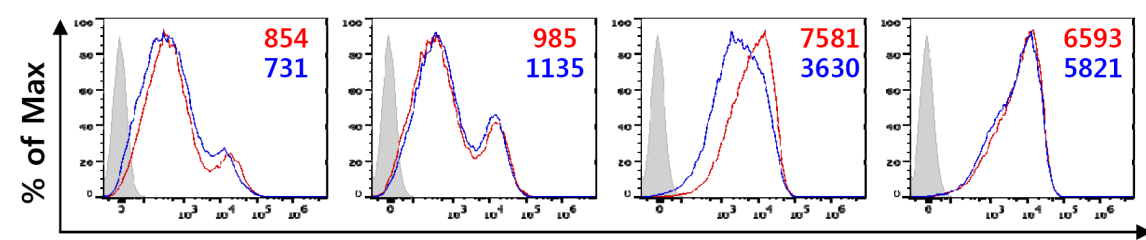

CD86

E

\section{F}

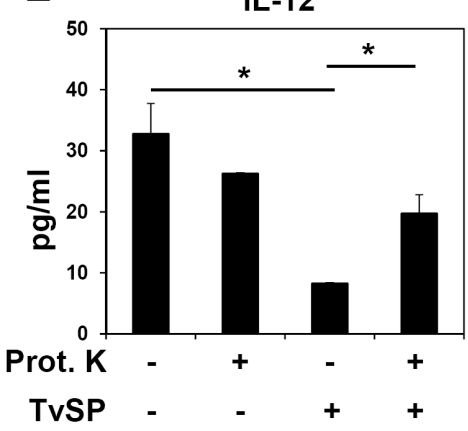

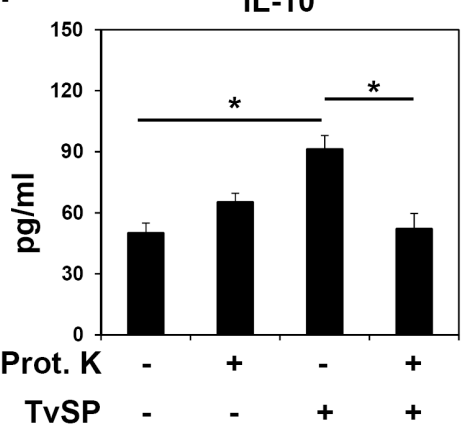

Fig. 3. The DC-modulatory activity of TvSP is abolished by treatment with Proteinase K. DCs were differentiated with GM-CSF from BM precursors in the presence of mock-treated or Proteinase K-treated TvSP. (A-D) On day 10 , DCs were treated with $100 \mathrm{ng} / \mathrm{ml}$ LPS for $24 \mathrm{~h}$ to induce maturation, and expression of MHC-II (A), CD40 (B), CD80 (C) and CD86 (D) was analyzed by flow cytometry. Isotype controls are shown as shaded histograms, control BMDCs are shown as red lines, and TvSP-treated BMDCs are shown as blue lines. Mean fluorescent intensity (MFI) values are indicated with corresponding colors. Results are representative of more than three independent experiments. (E, F) Production of cytokines IL-12 (E) and IL-10 (F) induced by LPS $(100 \mathrm{ng} / \mathrm{ml})$ treatment of BMDCs for $24 \mathrm{~h}$ as measured by cytometric bead array. All data are expressed as the means $\pm \mathrm{SD}(\mathrm{n}=3)$, and statistical significance $(* P<0.05)$ is shown for treatments compared with the controls. suggest that $T$. vaginalis can interfere with DC function without the need for direct cell-to-cell contact, by promoting immune tolerance and immune escape. First, TvSP can impair the expression of several surface molecules that play main roles in co-stimulation and antigen presentation to T cells. During differentiation, DCs up-regulate the expression of MHC-II and co-stimulatory molecules such as CD40, CD80, and CD86 and thus increase their efficiency as antigen-presenting cells. BMDCs differentiated in the presence of TvSP exhibited a significant defect in the expression of MHC-II and co-stimulatory molecules especially when the BMDCs were activated by LPS (Fig. 1). Second, BMDCs differentiated in the presence of TvSP 

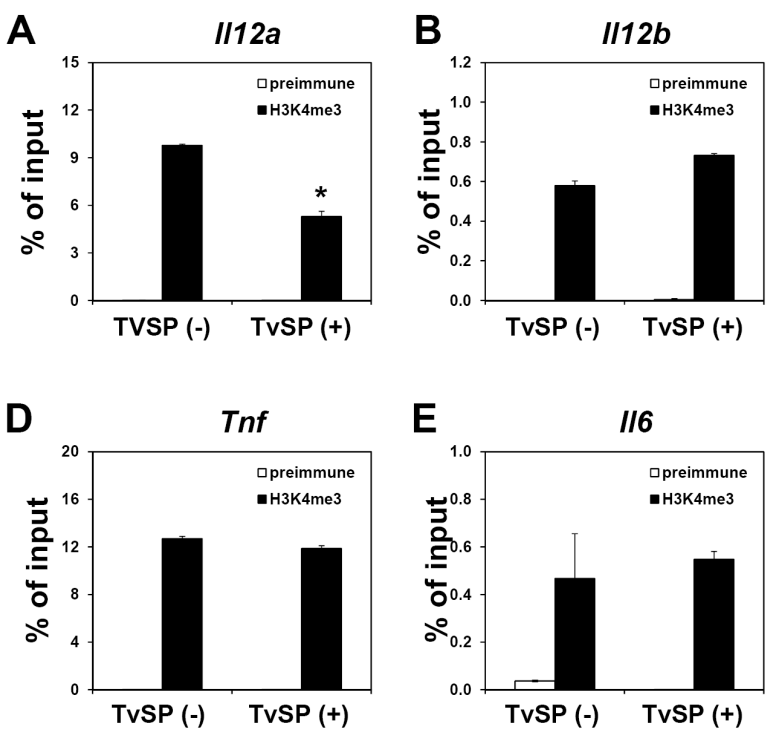

showed an altered cytokine production profile in response to Toll-like receptor 4 (TLR4)-mediated LPS signaling, as a marked reduction in pro-inflammatory cytokine IL-12 secretion and enhanced production of anti-inflammatory cytokine IL-10 were observed upon stimulation with LPS (Fig. 2). Interestingly, the expression of other pro-inflammatory cytokines such as TNF- $\alpha$ and IL- 6 was comparable between BMDCs differentiated in the absence or presence of TvSP, demonstrating that there is a separate regulation mechanism involved in the expression of each pro-inflammatory cytokine. Although it was reported that infection with $T$. vaginalis induced apoptosis of macrophages (18), the inhibition of DC maturation and altered cytokine production profiles in TvSP- treated BMDCs was not due to cytotoxicity because TvSP did not trigger significant apoptosis in BMDCs (Supplementary Fig. 1). These data suggest that $T$. vaginalis, via soluble products released during chronic infection, has the ability to shift an aggressive immune environment toward a more permissive one to promote its survival.

Treatment of TvSP with Proteinase $\mathrm{K}$ abolished its effect on DC including down-regulation of surface molecules and IL-12 production as well as up-regulation of IL-10 production (Fig. 3), suggesting that a proteinaceous TvSP component was necessary. However, we cannot entirely exclude contributions by any non-protein immunomodulatory components of TvSP, which might be thermally unstable. The identification of DC modulators secreted from $T$. vaginalis is an area for future investigation. The profiles of ERK, p38 MAPK, JNK, and NF-kB activation after LPS treatment is normal in BMDCs differentiated in the presence of TvSP (Supplementary Fig. 2), implying that TVSP may modulate the activity of BMDCs via distinct kinase pathways that have yet to be determined.

Epigenetic control and associated chromatin structure are
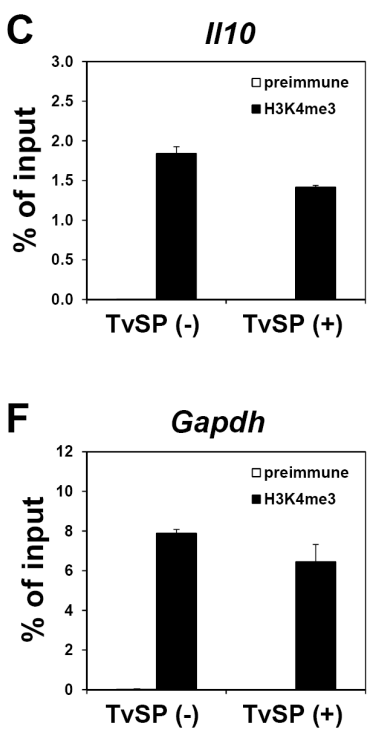

Fig. 4. TvSP exhibit epigenetic control via modulation of histone modifications at the promoter region of the I/12a gene. DCs were differentiated with GM-CSF from BM precursors in the absence or presence of TvSP. On day 10 , LPS $(100 \mathrm{ng} / \mathrm{ml})$ was added to the culture for $1 \mathrm{~h}$ and H3K4me3 levels at the promoter regions of $1 / 12 \mathrm{a}$ (A), $/ / 12 b(\mathrm{~B}), \quad / / 10(\mathrm{C}), \operatorname{Tnf}(\mathrm{D}), \quad / / 6(\mathrm{E})$ and Gapdh (F) genes were analyzed by ChIP assay. $* P<0.05$ compared with untreated cells. Results are representative of more than three independent experiments.

particularly significant contributors to gene regulation and therefore play major roles in determining and maintaining cell differentiation (24). Various types of posttranslational histone modifications, including acetylation and methylation, dictate the dynamic transition between transcriptionally active or silent chromatin structures (25). To determine whether histone methylation plays a role in dysregulated cytokine gene expression in TvSP-treated BMDCs, we performed a ChIP assay using an antibody against H3K4me3, a stable "mark" associated with actively-transcribed chromatin. As shown in Fig. 4A, the level of $\mathrm{H} 3 \mathrm{~K} 4 \mathrm{me} 3$ in the $1 / 12 \mathrm{a}$ promoter was significantly reduced in TvSP-treated BMDCs, suggesting that the down-regulation of I/12a gene expression in these cells is associated with transcriptionally less permissive histone modifications. In contrast, TvSP had no effect on enrichment of H3K4me3 in other promoters including $I / 12 b$ and $I / 10$ genes. How down-regulation of $\| 12 b$ and up-regulation of $\| 110$ are regulated by TvSP during BMDC differentiation remains to be investigated.

The data presented here demonstrate that soluble factors released by $T$. vaginalis, via a mostly proteinaceous component, control the LPS-induced maturation of BMDCs, resulting in a reduced production of IL-12 through an epigenetic mechanism. Considering the importance of IL-12 and IL-10 as well as the full activation of DC to efficiently prime T cells in the immune response against $T$. vaginalis, the ability of $T$. vaginalis to modulate these specific functions appears to be an effective immune evasion strategy.

\section{MATERIALS AND METHODS}

Detailed experimental procedures are described in online Supplementary information. 


\section{ACKNOWLEDGEMENTS}

This work was supported by National Research Foundation of Korea (NRF) grants funded by the Korean government (MSIP) (numbers; NRF-2011-0030086, 2012M3A9B4028272, and NRF-2013R1A1A2008812 to H.-P. Kim), and a faculty research grant from Yonsei University College of Medicine (6-20130156 to H.-P. Kim).

\section{REFERENCES}

1. Steinman RM (2012) Decisions about dendritic cells: past, present, and future. Annu Rev Immunol 30, 1-22

2. Banchereau J, Briere F, Caux C et al (2000) Immunobiology of dendritic cells. Annu Rev Immunol 18, 767-811

3. Morelli AE and Thomson AW (2007) Tolerogenic dendritic cells and the quest for transplant tolerance. Nat Rev Immunol 7, 610-621

4. Terrazas CA, Terrazas LI and Gomez-Garcia L (2010) Modulation of dendritic cell responses by parasites: a common strategy to survive. J Biomed Biotechnol 2010, 357106

5. Petrin D, Delgaty K, Bhatt R and Garber G (1998) Clinical and microbiological aspects of Trichomonas vaginalis. Clin Microbiol Rev 11, 300-317

6. Gerbase AC, Rowley JT, Heymann DH, Berkley SF and Piot P (1998) Global prevalence and incidence estimates of selected curable STDs. Sex Transm Infect 74 Suppl 1, S12-16

7. Landers DV, Wiesenfeld HC, Heine RP, Krohn MA and Hillier SL (2004) Predictive value of the clinical diagnosis of lower genital tract infection in women. Am J Obstet Gynecol 190, 1004-1010

8. Cotch MF, Pastorek JG 2nd, Nugent RP et al (1997) Trichomonas vaginalis associated with low birth weight and preterm delivery. The Vaginal Infections and Prematurity Study Group. Sex Transm Dis 24, 353-360

9. Kharsany AB, Hoosen AA, Moodley J, Bagaratee J and Gouws E (1993) The association between sexually transmitted pathogens and cervical intra-epithelial neoplasia in a developing community. Genitourin Med 69, 357-360

10. Shafir SC, Sorvillo FJ and Smith L (2009) Current issues and considerations regarding trichomoniasis and human immunodeficiency virus in African-Americans. Clin Microbiol Rev 22, 37-45

11. Kiviat NB, Paavonen JA, Brockway J et al (1985) Cytologic manifestations of cervical and vaginal infections. I. Epithelial and inflammatory cellular changes. JAMA 253, 989-996

12. Sardana S, Sodhani P, Agarwal SS et al (1994) Epidemiologic analysis of Trichomonas vaginalis infection in inflammatory smears. Acta cytologica 38, 693-697

13. Fouts AC and Kraus SJ (1980) Trichomonas vaginalis: reevaluation of its clinical presentation and laboratory diagnosis. J Infect Dis 141, 137-143

14. Ryu JS, Kang JH, Jung SY et al (2004) Production of interleukin-8 by human neutrophils stimulated with Tricho- monas vaginalis. Infect Immun 72, 1326-1332

15. Han IH, Goo SY, Park SJ et al (2009) Proinflammatory cytokine and nitric oxide production by human macrophages stimulated with Trichomonas vaginalis. Korean J Parasitol 47, 205-212

16. Chang JH, Ryang YS, Morio T, Lee SK and Chang EJ (2004) Trichomonas vaginalis inhibits proinflammatory cytokine production in macrophages by suppressing NF-kappaB activation. Mol Cells 18, 177-185

17. Scott K, Manunta M, Germain C et al (2005) Qualitatively distinct patterns of cytokines are released by human dendritic cells in response to different pathogens. Immunology $116,245-254$

18. Chang JH, Kim SK, Choi IH, Lee SK, Morio T and Chang EJ (2006) Apoptosis of macrophages induced by Trichomonas vaginalis through the phosphorylation of p38 mitogen-activated protein kinase that locates at downstream of mitochondria-dependent caspase activation. Int J Biochem Cell Biol 38, 638-647

19. Nam YH, Min A, Kim SH et al (2012) Leukotriene B(4) receptors BLT1 and BLT2 are involved in interleukin-8 production in human neutrophils induced by Trichomonas vaginalis-derived secretory products. Inflamm Res 61, 97-102

20. Nam YH, Min D, Kim HP et al (2011) Leukotriene B4 receptor BLT-mediated phosphorylation of NF-kappaB and CREB is involved in IL-8 production in human mast cells induced by Trichomonas vaginalis-derived secretory products. Microbes Infect 13, 1211-1220

21. Cobb MH and Goldsmith EJ (1995) How MAP kinases are regulated. J Biol Chem 270, 14843-14846

22. Rescigno M, Martino M, Sutherland CL, Gold MR and Ricciardi-Castagnoli P (1998) Dendritic cell survival and maturation are regulated by different signaling pathways. J Exp Med 188, 2175-2180

23. Sproul D, Gilbert N and Bickmore WA (2005) The role of chromatin structure in regulating the expression of clustered genes. Nat Rev Genet 6, 775-781

24. Kanno Y, Vahedi G, Hirahara K, Singleton K and O'Shea JJ (2012) Transcriptional and epigenetic control of $T$ helper cell specification: molecular mechanisms underlying commitment and plasticity. Annu Rev Immunol 30, 707-731

25. Fisher CL and Fisher AG (2011) Chromatin states in pluripotent, differentiated, and reprogrammed cells. Curr Opin Genet Dev 21, 140-146

26. Pereira-Neves A and Benchimol M (2007) Phagocytosis by Trichomonas vaginalis: new insights. Biol Cell 99, 87-101

27. Lutz MB, Kukutsch N, Ogilvie AL et al (1999) An advanced culture method for generating large quantities of highly pure dendritic cells from mouse bone marrow. J Immunol Methods 223, 77-92

28. Lee JJ, Park K, Shin MH et al (2011) Accessible chromatin structure permits factors Sp1 and Sp3 to regulate human TGFBI gene expression. Biochem Biophys Res Commun 409, 222-228

29. Park K, Park JH, Yang WJ, Lee JJ, Song MJ and Kim HP (2012) Transcriptional activation of the IL31 gene by NFAT and STAT6. J Leukoc Biol 91, 245-257 